\title{
Mitotic Rate and Younger Age Are Predictors of Sentinel Lymph Node Positivity: Lessons Learned From the Generation of a Probabilistic Model
}

\author{
Vernon K. Sondak, MD, Jeremy M. G. Taylor, PhD, Michael S. Sabel, MD, Yue Wang, PhD, \\ Lori Lowe, MD, Amelia C. Grover, MD, Alfred E. Chang, MD, Alan M. Yahanda, MD, \\ James Moon, MS, and Timothy M. Johnson, MD
}

\begin{abstract}
Background: Sentinel lymph node (SLN) biopsy allows surgeons to identify patients with subclinical nodal involvement who may benefit from lymphadenectomy and, possibly, adjuvant therapy. Several factors have been variably, and sometimes discordantly, reported to have predictive value for SLN metastasis to best select which patients require SLN biopsy.

Methods: We reviewed 419 patients who underwent SLN biopsy for melanoma from a prospectively collected melanoma database. To derive a probabilistic model for the occurrence of a positive SLN, a multivariate logistic model was fit by using a stepwise variable selection method. The accuracy of each model was evaluated by using receiver operator characteristic curves.

Results: On univariate analysis, the number of mitoses per square millimeter, increasing Breslow depth, decreasing age, ulceration, and melanoma on the trunk showed a significant relationship to a positive SLN. Multivariate analysis revealed that once age, mitotic rate, and Breslow thickness were included, no other factor, including ulceration, was significantly associated with a positive SLN. The data suggest that younger patients with tumors $<1 \mathrm{~mm}$ may still have a substantial risk for a positive SLN, especially if the mitotic rate is high.

Conclusions: In addition to Breslow depth, mitoses per square millimeter and younger age were factors identified as independent predictors of a positive SLN. This model may identify patients with thin melanoma at sufficient risk for metastases to justify SLN biopsy.
\end{abstract}

Key Words: Melanoma-Sentinel lymph node-Mitotic rate-Ulceration.

One of the most significant advances in the management of primary cutaneous melanoma has been the concept of lymphatic mapping and sentinel lymph node (SLN) biopsy. Since the initial landmark publication by Morton et al. ${ }^{1}$ that applied the concept to melanoma only a decade ago, lymphatic mapping and SLN biopsy have evolved through refinement, clinical experience, and

Received March 7, 2003; accepted October 16, 2003.

From the Departments of Surgery (VKS, MSS, ACG, AEC, AMY, TMJ), Biostatistics (JMGT, YW, JM), Pathology (LL), Dermatology (LL, TMJ), and Otolaryngology (TMJ), University of Michigan Health System, Ann Arbor, Michigan.

Address correspondence and reprint requests to: Vernon K. Sondak, MD, Room 3306, Cancer Center, 1500 East Medical Center Drive, Ann Arbor, MI 48109-0932; Fax: 734-647-9647; E-mail: vsondak@ umich.edu.

Published by Lippincott Williams \& Wilkins @ 2004 The Society of Surgical Oncology, Inc. clinical trials to become an invaluable technique for identifying patients who harbor subclinical lymph node metastasis, with minimal morbidity. ${ }^{1-7}$ By documenting those with subclinical nodal involvement, surgeons are now able to identify patients who may benefit from lymphadenectomy and possibly adjuvant therapy while sparing patients with negative SLN biopsy results from the morbidity associated with these treatments. Furthermore, the single most important factor to date that determines overall prognosis and survival for stage I and II melanoma is the SLN status. ${ }^{1-7}$

SLN biopsy is widely accepted as a means of staging the lymph nodes for melanoma and is practiced as standard care at virtually all major melanoma centers. ${ }^{6}$ Breslow thickness (measured in millimeters) is the only reproducible factor predictive of SLN status in all studies. ${ }^{1-3,7-23}$ Several other factors have been variably and 
sometimes discordantly reported to have predictive value for SLN metastasis, including Clark level, ulceration, mitotic rate, microsatellitosis, angiolymphatic invasion, vertical growth phase, number of nodal basins, primary tumor location, and patient age. ${ }^{1-3,7-23}$ Several plausible explanations could account for the disparate and sometimes conflicting predictive models that have been offered to date. Foremost among them is the use of different sets of predictors. Few studies involve all known predictive factors, so no two models start with identical sets of possible predictors. Less readily recognized-but no less significant-is the fact that many different combinations of predictors can yield a model that has a strong correlation with SLN status. Unless different models are compared directly by using the same data set, there is no way to determine how well a particular model performs.

For this study, we reviewed a prospectively collected comprehensive melanoma database and identified 429 consecutive patients who underwent SLN biopsy at a single institution. The primary objective was to assess the factors that most strongly influence the probability of finding a positive SLN in patients with cutaneous melanoma. Secondary objectives included the following: to understand the effects of and interactions between multiple factors in a probabilistic model of SLN metastasis, to explore the degree to which any combination of available factors can fully predict the status of the SLNs, and to estimate whether any patient subgroups currently not routinely considered for SLN biopsy are in fact at a high-enough risk of having nodal metastasis to justify prospectively evaluating extended indications for SLN biopsy.

\section{METHODS}

Approval of the study was granted by the University of Michigan Institutional Review Board for Medicine. Our prospective melanoma database was queried for patients entered from January 1996 to August 1999 to identify consecutive patients who underwent lymphatic mapping and SLN biopsy. Patients were grouped by the presence or absence of metastatic melanoma in at least one SLN; thus, patients with one or more positive nodes were considered as having a positive SLN biopsy.

\section{Histological Features}

The histological features analyzed were Breslow thickness (measured in millimeters), deep margin status (to identify incisional or shave biopsies), growth phase (vertical vs. radial), and the number of mitoses per square millimeter $\left(1 \mathrm{~mm}^{2}\right.$ is approximately equal to four to five high-power $[\times 40]$ microscope fields, counted beginning in the fields with the most mitoses), as well as the presence or absence of the following: angiolymphatic invasion, regression, microsatellitosis, ulceration, neurotropism, and periadnexal extension. The site of the primary melanoma was also evaluated and categorized into one of four anatomical locations: head and neck, trunk, upper extremity, or lower extremity. Patients with a melanoma located on any mucous membrane or on the genitalia were excluded from the analysis. In addition, the effects of age and sex were also considered. All primary lesions were evaluated prospectively and without knowledge of the subsequent status of the SLN biopsy by a dermatopathologist with expertise in melanoma by using a comprehensive 14-point melanoma profile generated for every primary lesion.

Two more variables were created from the existing database to compare this analysis with one previously performed and reported by Mraz-Gernhard et al. ${ }^{8}$ The first was a high mitotic rate, defined by those authors as more than five mitoses per square millimeter. The other was the total number of high-risk factors, which they defined as the number of the following factors present in a given lesion: ulceration, high mitotic rate, microsatellitosis, angiolymphatic spread, vertical growth phase, and regression (but not Breslow depth).

\section{SLN Biopsy Technique}

In general, patients with lesions $\geq 1.0 \mathrm{~mm}$ in Breslow depth or $<1.0 \mathrm{~mm}$ with at least one adverse histopathologic feature (ulceration, positive deep margin, or significant regression) were considered for lymphatic mapping and SLN biopsy. Clark level was not used as an indication for SLN biopsy of thin lesions. Patients with a previous wide excision $(>1-\mathrm{cm}$ margin) and those with significant comorbidities were not considered good candidates for lymphatic mapping and SLN biopsy and were evaluated for biopsy on an individualized basis but were not arbitrarily excluded. All patients underwent sameday preoperative lymphoscintigraphy in which ${ }^{99 m} \mathrm{Tc}$ sulfur colloid (CIS-US Inc., Bedford, MA) was injected intradermally around the primary lesion or biopsy site 2 to 4 hours before surgery to identify lymphatic basins and in-transit nodes at risk. Patients in whom the lymphoscintigram showed no obvious transit of radionuclide to any nodal basin were evaluated during surgery, and an attempt was made to locate the SLN by using a handheld gamma probe and blue dye. Patients in whom there was transit to multiple basins were approached selectively, and as many as four but generally no more than two basins were subjected to biopsy. After induction of satisfactory anesthesia (usually general inhaled anesthesia 
administered by laryngeal mask or endotracheal tube), isosulfan blue dye (Lymphazurin 1\%; Hirsch Industries Inc., Richmond, VA) was injected intradermally around the primary lesion or narrow excision biopsy site, and the area was massaged to promote lymphatic flow. After approximately 5 to 10 minutes, a handheld gamma probe (Navigator GPS; US Surgical, Cincinnati, OH) was used to identify "hot" spots over the SLNs. A small incision was made directly overlying the hot spots and carried down through the skin and subcutaneous tissue into the node-bearing fat. Any blue-stained, hot (defined as the hottest node and any other nodes within $10 \%$ of the counts per minute of the hottest node), or palpably abnormal nodes were considered SLNs. Once identified, the SLNs were excised and sent for careful histopathologic analysis. Definitive wide excision of the primary cutaneous melanoma was then performed. SLNs were evaluated by one of two dermatopathologists with experience and expertise in melanoma and SLN evaluation. SLNs were serially sectioned and stained with hematoxylin and eosin (H\&E). H\&E-negative nodes were further stained with a battery of immunohistochemistry stains, most often consisting of S-100, Melan-A, and HMB-45, as previously described. ${ }^{24}$ Reverse transcriptase-polymerase chain reaction was not used to evaluate any SLNs.

\section{Statistical Analysis}

The event of interest was defined as the presence of one or more H\&E- or immunohistochemistry-positive SLNs discovered during any SLN biopsy on a particular patient. To determine the association between each clinical or pathologic factor and the event of interest, a logistic regression model was fit with each factor by itself and each factor adjusted for the age and sex of the patient. By using the parameter estimates from these models, the $P$ value from the Wald $\chi^{2}$ test for the significance of the parameter, the odds ratio, and a $95 \%$ Wald-based confidence interval for the odds ratio were reported. We do not undertake any formal adjustment of $P$ values, even though multiple tests were performed. Because of the exploratory nature of the search for variables that were associated with SLN positivity, the reader should be cautious not to overinterpret the importance of variables that have marginally significant associations.

To derive a probabilistic model for the occurrence of one or more positive SLNs, a multivariate logistic model was fit by using a stepwise variable selection method. Factors were entered into the model if they were independently significant at the .10 level but were dropped if they were not significant at the .05 level when adjusted for variables already in the model. To compare the ef- fects when adjusted for age and sex and to allow comparison with other published data, a second multivariate model was fit in the same stepwise fashion, only this time age and sex were forced into one model. Finally, a model containing all two-way interactions between significant variables was considered. The analysis was performed with SAS PROC LOGISTIC (version 6.12; SAS Institute, Cary, NC), which discards an entire record if it contains a missing value for either the outcome variable or one of the variables in the model.

The accuracy of each model was evaluated by using receiver operator characteristic (ROC) curves. The ROC is a plot of $(1-$ specificity $)$ versus sensitivity and is frequently used to evaluate diagnostic procedures. For an ideal predictive test, both the sensitivity and specificity should be high, as occurs when the ROC curve is shaped like an inverted $\mathrm{L}$, with its corner close to the top left corner of the plot. Conversely, a test with only a random relationship to the outcome- such as the toss of a coinwould be a straight line at a $45^{\circ}$ angle from the lower left to upper right. For two tests of less-than-perfect accuracy, the one whose ROC curve is closer to the idealized L-shaped plot is the more accurate.

The linear combinations of factors derived in the logistic regression model generate a risk score that is converted into a probability of a positive SLN by using the equation $P=$ $\exp (\mathrm{RS}) /[1+\exp (\mathrm{RS})]$, where $P$ is the probability of SLN positivity and RS stands for risk score and is a linear combination of the chosen covariates.

A decision rule is constructed to categorize the patient's SLN as predicted to be positive if the probability is greater than a given cutoff and is predicted to be negative otherwise. For each possible cutoff value, the sensitivity of the rule is the fraction of correct calls among the true positives, and the specificity is the fraction of correct calls among the true negatives. As the cutoff is varied, the sensitivity and specificity change and are plotted in the ROC curve. Two ROC curves can be constructed for a given model: one for which all the data are resubstituted into the equation and the other with leave-one-out cross-validation. The cross-validated ROC curve is generally considered the more legitimate estimate and is the one presented.

\section{RESULTS}

There were 429 consecutive patients identified in the database (248 men and 181 women) during the 3.5-year study. Of these, 419 patients $(97.7 \%)$ had an identified and evaluated SLN and available data, and they form the basis of this report. Thirty-one patients underwent simultaneous SLN biopsies from two or three different nodal 
basins (primary lesion location with more than one primary nodal basin, such as the trunk), and these patients were considered positive if at least one biopsy in one or more basins identified a positive SLN. There were 416 patients for whom the site of the primary melanoma was available. These broke down in the following manner: 58 were located on the head or neck, 187 were located on the trunk, 69 were located on an upper extremity, and 102 were located on a lower extremity. One or more histologically or immunohistochemically positive lymph nodes were found in $76(18.1 \%)$ of 419 patients.

Table 1 consists of descriptive statistics for each of the histological and clinical factors considered, including a breakdown of the SLN biopsy results for the patients who had each factor. The unadjusted percentage of pos-

TABLE 1. Sentinel lymph node (SLN) biopsy results for each of the histological and clinical factors considered

\begin{tabular}{|c|c|c|}
\hline Variable & No. Present & $\%$ Positive SLNs \\
\hline \multicolumn{3}{|l|}{ Sex } \\
\hline Male & 243 & 18.9 \\
\hline Female & 176 & 16.5 \\
\hline \multicolumn{3}{|l|}{ Age (y) } \\
\hline$<35$ & 76 & 26.3 \\
\hline $35-60$ & 215 & 18.6 \\
\hline$>60$ & 127 & 11.8 \\
\hline \multicolumn{3}{|l|}{ Primary tumor site } \\
\hline Head and neck & 57 & 12.3 \\
\hline Trunk & 184 & 23.4 \\
\hline Upper extremity & 68 & 7.4 \\
\hline Lower extremity & 98 & 19.4 \\
\hline \multicolumn{3}{|l|}{ Breslow depth (mm) } \\
\hline$<1.0$ & 42 & 9.5 \\
\hline $1.0-2.0$ & 217 & 12.9 \\
\hline $2.0-4.0$ & 126 & 23.0 \\
\hline$>4.0$ & 31 & 45.2 \\
\hline \multicolumn{3}{|l|}{ Growth phase } \\
\hline Radial growth only & 2 & 0 \\
\hline Vertical growth or unknown & 407 & 18.2 \\
\hline \multicolumn{3}{|l|}{ Mitotic rate $\left(\right.$ per $\left.\mathrm{mm}^{2}\right)$} \\
\hline$<1$ & 271 & 12.2 \\
\hline $1-5$ & 109 & 27.5 \\
\hline$>5$ & 24 & 41.7 \\
\hline \multicolumn{3}{|l|}{ Ulceration } \\
\hline Absent & 317 & 15.8 \\
\hline Present & 96 & 25.0 \\
\hline \multicolumn{3}{|l|}{ Angiolymphatic invasion } \\
\hline Absent & 395 & 17.2 \\
\hline Present & 18 & 33.3 \\
\hline \multicolumn{3}{|l|}{ Regression } \\
\hline Absent & 346 & 18.2 \\
\hline Present & 67 & 16.4 \\
\hline \multicolumn{3}{|l|}{ Neurotropism } \\
\hline Absent & 400 & 17.5 \\
\hline Present & 13 & 30.8 \\
\hline \multicolumn{3}{|l|}{ Microsatellitosis } \\
\hline Absent & 399 & 17.5 \\
\hline Present & 14 & 28.6 \\
\hline \multicolumn{3}{|l|}{ Periadnexal extension } \\
\hline Absent & 412 & 18.0 \\
\hline Present & 4 & 25.0 \\
\hline
\end{tabular}

itive SLN biopsies was slightly higher for men than women and for primary tumors of the trunk and lower extremity. The percentage of positive biopsies increased with increasing tumor thickness and with increases in the number of mitoses per square millimeter (mitotic rate). The percentage of positive SLN biopsies was higher for those primary tumors with any adverse histological features except regression. Patients ranged in age from 9 to 80 years old and were arbitrarily divided into three age groups: $<35,35$ to 60 , and $>60$ years of age. It is interesting to note that the percentage of node-positive biopsies was highest for the youngest age group $(<35$ years; $26.3 \%$ positive) and was progressively lower for the older groups (35 to 60 years, $18.6 \%$ positive; $>60$ years, $11.8 \%$ positive).

Table 2 summarizes the results of univariate analyses of the relationships described previously, for each factor by itself and when adjusted for age and sex. When considered alone, the number of mitoses per square millimeter $(P<.0001)$, increasing Breslow depth $(P<$ $.0001)$, decreasing age $(P=.003)$, presence of ulceration $(P=.04)$, and having a primary melanoma located on the trunk $(P=.01)$ or upper extremity $(P=.02$, inverse correlation) showed a significant relationship with the incidence of one or more positive SLNs. Angiolymphatic spread showed a marginally significant association $(P=$ .09). In addition, the presence of increasing numbers of high-risk factors as described by Mraz-Gernhard et al. ${ }^{8}$ was strongly associated with the incidence of positive SLNs $(P=.0009)$. When adjusted for age and sex, the results were nearly identical. The only exception was that an upper extremity primary tumor site was no longer associated with a statistically significant decrease in the probability of a positive node (adjusted $P=.06$ ).

Multivariate analysis revealed that once age, number of mitoses per square millimeter, and Breslow thickness were included, no other factor was significantly associated with the incidence of a positive SLN. Forcing the addition of sex to the multivariate model resulted in identical results. Thus, the results of multivariate analysis indicate that once age, Breslow thickness, and mitotic rate are accounted for, no other prognostic feature (including ulceration) adds independent predictive value. Of note, however, if we had not included mitotic rate in our model, then the odds ratio estimate for ulceration would be 2.01 (95\% confidence interval, 1.14-3.54; $P=$ .02). With age, ulceration, and mitotic rate, the odds ratio for ulceration is 1.48 (95\% confidence interval, .81-2.70; $P=.21)$.

Testing for interaction among the predictive variables indicated a complex relationship between age, thickness, and mitotic rate and the probability of a positive biopsy. 
TABLE 2. Univariate analyses of the relationships between the histological and clinical factors considered, for each factor by itself and when adjusted for age and sex

\begin{tabular}{|c|c|c|c|}
\hline Factor & $P$ value & OR & $95 \% \mathrm{CI}$ \\
\hline \multicolumn{4}{|l|}{ Univariate analysis } \\
\hline Angiolymphatic spread & .09 & 2.40 & $.87-6.63$ \\
\hline Regression & .73 & .88 & $.43-1.78$ \\
\hline Number of mitoses $/ \mathrm{mm}^{2}$ & $<.0001$ & 1.26 & $1.13-1.41$ \\
\hline High mitotic rate $\left(>5 / \mathrm{mm}^{2}\right)$ & .003 & 3.59 & $1.52-8.45$ \\
\hline Vertical growth phase $^{a}$ & .56 & .79 & $.34-1.78$ \\
\hline Satellitosis & .30 & 1.88 & $.57-6.17$ \\
\hline Ulceration & .04 & 1.78 & $1.03-3.09$ \\
\hline Neurotropism & .23 & 2.10 & $.63-7.00$ \\
\hline Periadnexal extension present & .72 & 1.52 & $.16-14.8$ \\
\hline Breslow depth & $<.0001$ & 1.31 & $1.15-1.50$ \\
\hline Head and neck location & .23 & .60 & $.26-1.38$ \\
\hline Trunk location & .01 & 1.92 & $1.16-3.18$ \\
\hline Upper extremity location & .02 & .32 & $.12-.82$ \\
\hline Lower extremity location & .68 & 1.13 & $.63-2.01$ \\
\hline Sex & .52 & 1.18 & $.71-1.97$ \\
\hline Age & .003 & .98 & $.96-.99$ \\
\hline High-risk factors ${ }^{b}$ & .0009 & 1.85 & $1.28-2.65$ \\
\hline \multicolumn{4}{|c|}{ Univariate analysis with adjustment for age and sex } \\
\hline Angiolymphatic spread & .06 & 2.67 & $.94-7.56$ \\
\hline Regression & .73 & .88 & $.43-1.80$ \\
\hline Number of mitoses $/ \mathrm{mm}^{2}$ & $<.0001$ & 1.27 & $.14-1.42$ \\
\hline High mitotic rate $\left(>5 / \mathrm{mm}^{2}\right)$ & .005 & 3.53 & $1.47-8.47$ \\
\hline Vertical growth phase $^{a}$ & .53 & .76 & $.33-1.77$ \\
\hline Satellitosis & .22 & 2.18 & $.63-7.58$ \\
\hline Ulceration & .02 & 1.93 & $1.09-3.43$ \\
\hline Neurotropism & .11 & 2.74 & $.80-9.41$ \\
\hline Periadnexal extension present & .70 & 1.58 & $.16-16.1$ \\
\hline Breslow depth & $<.0001$ & 1.32 & $1.15-1.51$ \\
\hline Head and neck location & .30 & .64 & $.27-1.50$ \\
\hline Trunk location & .04 & 1.72 & $1.02-2.87$ \\
\hline Upper extremity location & .06 & .39 & $.15-1.03$ \\
\hline Lower extremity location & .82 & 1.07 & $.59-1.95$ \\
\hline High-risk factors ${ }^{b}$ & .0005 & 1.96 & $1.34-2.86$ \\
\hline
\end{tabular}

OR, odds ratio; $\mathrm{CI}$, confidence interval.

${ }^{a}$ Subjects with unknown growth phase were not counted as vertical growth phase.

${ }^{b}$ High-risk factors were ulceration, microsatellites, angiolymphatic invasion, and high mitotic rate (more than five mitoses per square millimeter).

There was a significant interaction between Breslow thickness and mitotic rate $(P=.01)$, Breslow thickness and age $(P=.02)$, and age and mitotic rate $(P=.01)$ on the probability of SLN positivity.

The results of the multivariate analysis led to the construction of a probabilistic model by using the equation described in Methods. The final model contains Breslow depth, number of mitoses, age, and all two-way interactions between them, where the value for the risk score (RS) in the equation is calculated by the formula:

$$
\begin{aligned}
\mathrm{RS}= & -.8832-.0387 \text { (age) }+1.2042 \text { (mitoses) } \\
& -.2862(\text { Breslow })-.0165 \text { (age mitosis }) \\
& +.0131 \text { (age Breslow) }-.0509 \text { (mitosis Breslow) }
\end{aligned}
$$

The results of the multivariate analysis can be expressed graphically by using logistic regression analysis that displays the predicted probability of a positive SLN biopsy when two of the three factors are fixed. Figure 1 shows probability plots over the range of each of the factors included in the final model when the other two factors are fixed. Thus, for a fixed age and mitotic rate (Fig. 1A), the predicted probability of a positive biopsy increases as the Breslow thickness of the tumor increases. At a fixed thickness and age, the predicted probability increases as the number of mitoses per square millimeter increases (Fig. 1B). Finally, at a fixed thickness and mitotic rate, the likelihood of a positive biopsy decreases with increasing age (Fig. 1C).

The effect of this interaction is shown graphically in Fig. 2, which demonstrates the interaction between age and tumor thickness when the mitotic rate is held constant. For younger patients (age 35 in this illustration, in which only four specific ages are shown for clarity of presentation), the likelihood of a positive biopsy is relatively less dependent on tumor thickness, ranging from 

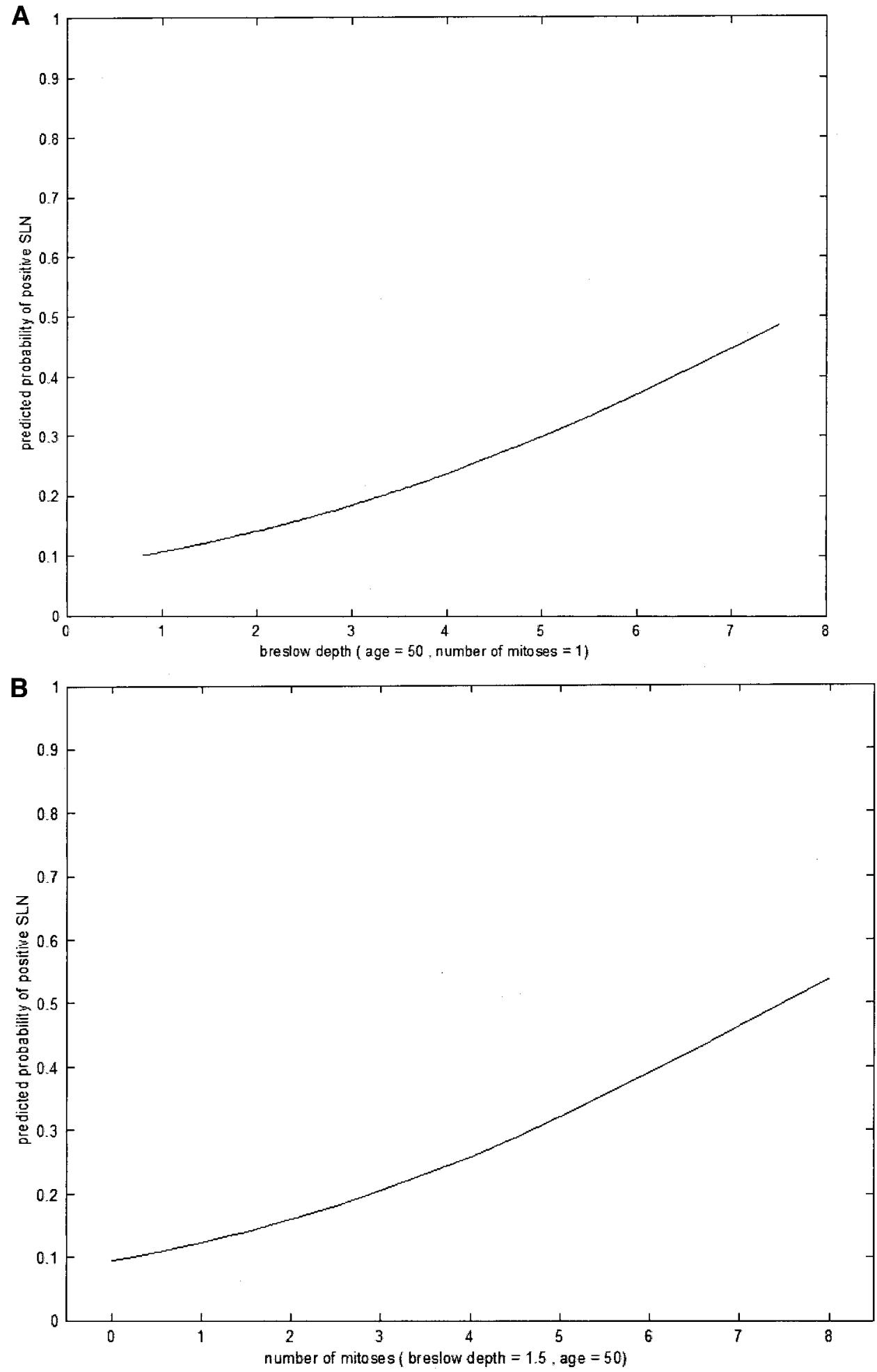


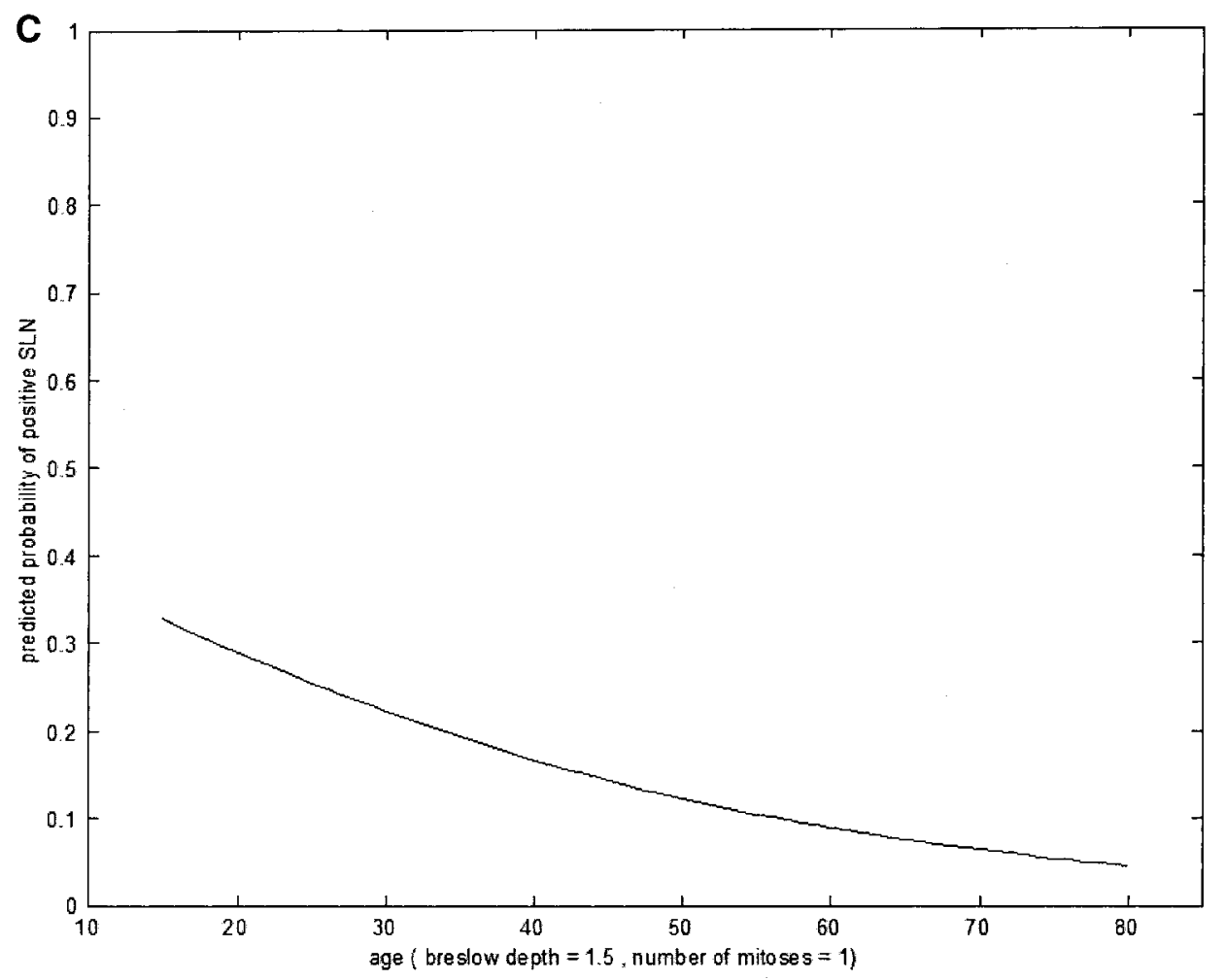

a predicted probability of just under $20 \%$ for a $1-\mathrm{mm}$ tumor that has one mitosis per square millimeter to slightly below $30 \%$ for a 6 -mm tumor with the same mitotic rate. By contrast, for older patients, the likelihood of a positive biopsy is far more dependent on thicknesswell under a $10 \%$ probability for a 65 -year-old patient with a 1-mm tumor and one mitosis per square millimeter to approximately $45 \%$ for a same-age patient with a 6-mm tumor and the same mitotic rate. Although the number of patients sampled with tumors $<1 \mathrm{~mm}$ thick was very small, the trend in Fig. 2 strongly suggests that patients $\leq 35$ years old who have tumors thinner than $1 \mathrm{~mm}$ may still have a substantial risk for having a positive SLN.

Figure 3 offers an alternate illustration of the varying effects of mitotic rate with age. In younger patients (the probability data for 35 years of age are shown in Fig. $3 \mathrm{~A}$ ), when the mitotic rate is high, there is a high probability of finding a positive SLN at all tumor thicknesses from 1 to $7 \mathrm{~mm}$. The relative flatness of the curves, especially for the highest mitotic rates illustrated, reflects the relative insensitivity of the probability prediction to changes in tumor thickness. In contrast, for a 50-year-old patient (Fig. 3B), the number of mitoses greatly affects the likelihood of finding a positive node for a fairly thin tumor but not for a very thick tumor. For a 65-year-old patient (data not shown), there is almost no effect of the number of mitoses on the likelihood of finding a positive node over almost the entire range of tumor thicknesses. This is nicely shown graphically by observing the decreasing effect of mitotic rate on the predicted probability of a positive node with increasing age for a $1.5-\mathrm{mm}$ thick tumor (Fig. 4).

As previously indicated, the overall accuracy of predictive models or tests can be displayed and compared by examining ROC curves. Figure 5 compares four validated ROC curves by using different predictive formulas. Breslow thickness alone has poor sensitivity and specificity-better than a coin flip but not by much. Adding

FIG. 1. Breslow depth, age, and mitotic rate are independent variables on multivariate analysis for predicting the presence of a positive sentinel lymph node (SLN). To derive a probabilistic model for the occurrence of one or more positive SLNs, a multivariate logistic model was fit by using a stepwise variable selection method. A probability plot was generated for each independent factor while the other factors were kept constant. (A) For a fixed age and mitotic rate (50 years old and one mitosis per square millimeter), the predicted probability of a positive SLN increases as the Breslow thickness of the tumor increases. At a fixed thickness and age (in this case, $1.5 \mathrm{~mm}$ and 50 years), the predicted probability increases as the number of mitoses per square millimeter increases $(\mathbf{B})$. At a fixed thickness and mitotic rate $(1.5 \mathrm{~mm}$ and one mitosis per square millimeter), the likelihood of a positive SLN decreases with increasing age (C). 


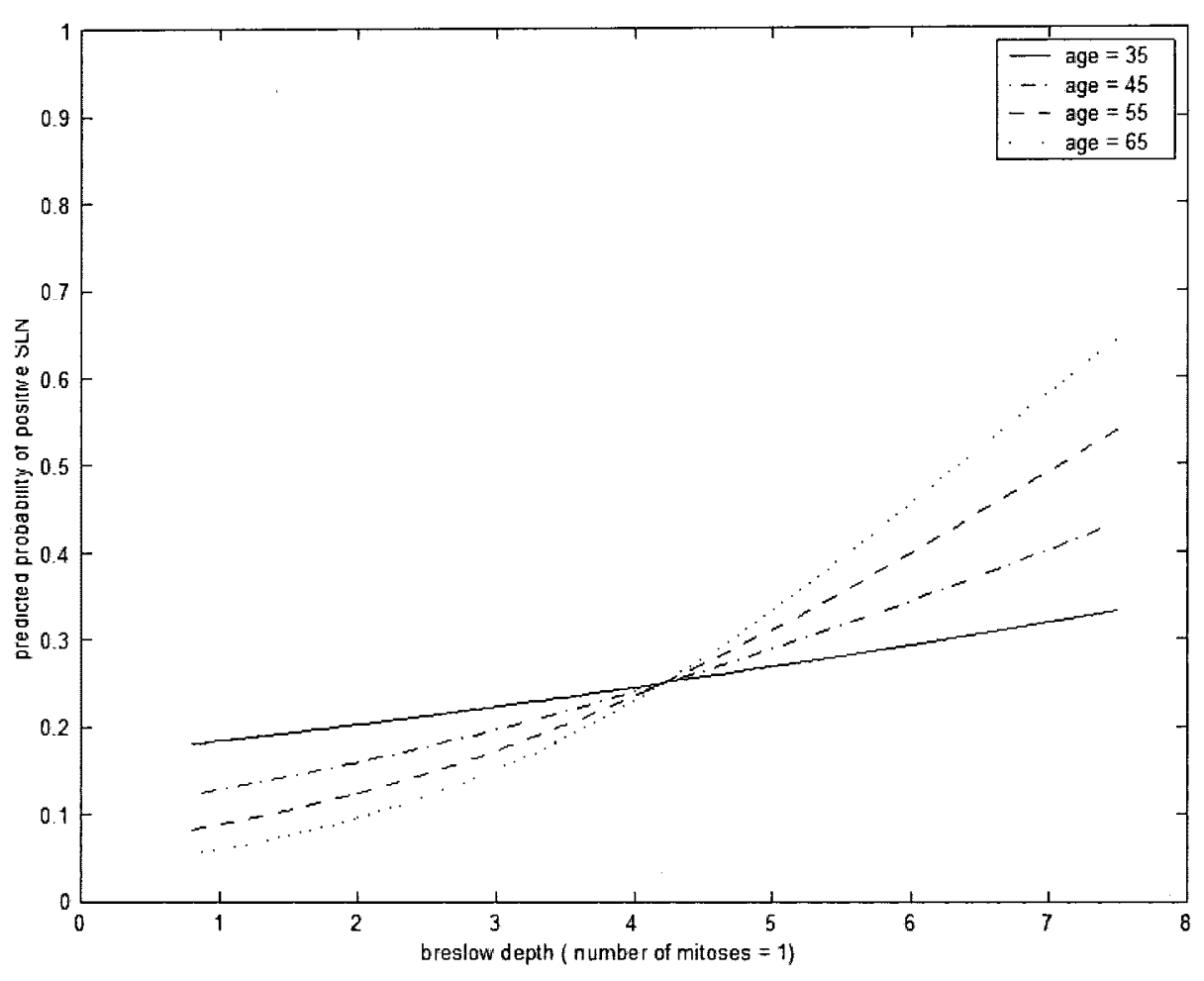

FIG. 2. The effect of Breslow thickness on sentinel lymph node (SLN) positivity is greater with increasing age. A probability plot for a positive SLN for increasing age and Breslow depth at a constant number of mitoses (one per square millimeter) demonstrates that Breslow depth has a more significant effect with increasing age (i.e., a steeper slope to the curves at older ages). ulceration to the Breslow thickness (as in the new American Joint Committee on Cancer [AJCC] staging system for melanoma) actually does little to increase the accuracy of prediction; the two curves overlap over virtually their entire course. In contrast, the ROC curve for the predictive model of Breslow thickness, age, and mitotic rate moves modestly up and to the left relative to the first two curves, indicating slightly greater sensitivity and specificity. To see whether accuracy could be improved further by adding more information to the predictive model, the fourth curve uses the total number of highrisk factors ${ }^{8}$ - ulceration, high mitotic rate, microsatellitosis, angiolymphatic spread, vertical growth phase, and regression-but does not improve the shape of the ROC curve at all. It is worth noting that the large area to the left and above the highest ROC curve represents the relatively large domain of predictive information that is not included in any of the predictive models tested.

\section{DISCUSSION}

The SLN biopsy represents one of the most significant advances in melanoma management over the past decade. It allows for selective application of therapeutic lymph node dissection only to node-positive patients with melanoma. As such, it maximizes the likelihood that a survival benefit will be realized with this ap- proach. ${ }^{15}$ However, demonstration of a survival benefit is not a requisite to justify the use of the procedure. SLN biopsy is a staging technique that is superior to any others yet identified. As long as the need for accurate staging of the regional nodes exists, the procedure is justified if it can be performed with high success rates, low false-negative rates, and minimal morbidity.

In our series, $18 \%$ of patients who underwent lymphatic mapping and SLN biopsy had one or more positive SLNs, a figure very consistent with other studies. Numerous studies have reported a number of clinical and histopathologic factors that correlate with the predictive probability of the SLN status. Results from these studies using variable statistical methods have yielded conflicting conclusions. The single uniform factor that correlates with SLN status is the Breslow thickness of the primary lesion, although uniform cutoffs for depth categories are missing from these studies. Collectively, however, as the Breslow depth increases, the probability of a positive SLN increases. Evaluating Breslow depth alone, for lesions $<1.0 \mathrm{~mm}$ in thickness, the published collective rate of SLN positivity is $<5 \%$. $2,7,8,13,16,18$ In lesions $>3.0$ $\mathrm{mm}$, the published rate of SLN positivity ranges from $20 \%$ to $50 \%$. $2,7-10,13,16-18,20,21,23$

Several reports have studied other primary lesion factors associated with SLN status. Breslow thickness is consistently reported in association with SLN status. 

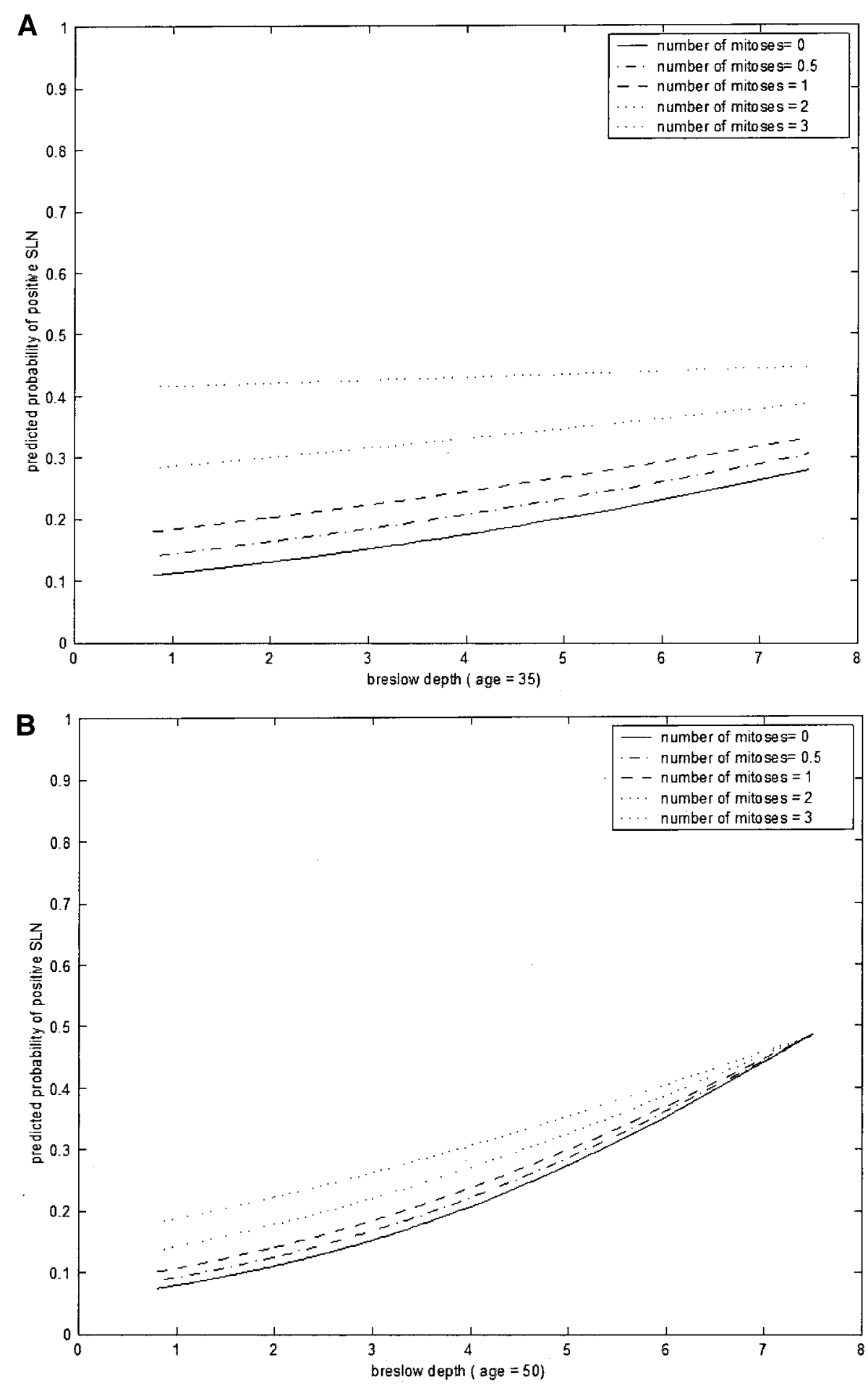

FIG. 3. Effect of Breslow thickness and mitotic rate at age 35 and age 50 . At age 35 (A), the effect of Breslow thickness on the likelihood of a positive sentinel lymph node (SLN) is most significant (i.e., the slope of the curve is steepest) when the mitotic rate is 0 (solid line). With increasing mitotic rate, the likelihood of a positive SLN increases (i.e., the entire curve rises), and the effect of depth on probability decreases (the slope falls). At a mitotic rate of three per square millimeter, the curve is nearly flat: Breslow depth has a minimal effect on the probability of a positive SLN, with a similar likelihood for both a 1-mm melanoma and a 7-mm melanoma. At age $50(\mathbf{B})$, the mitotic rate demonstrates less effect (less separation of the curves), and increasing Breslow thickness is the best predictor of a positive SLN (i.e., there is a steep slope for all curves, with convergence of the curves at high Breslow thicknesses).
Microsatellitosis, vascular invasion (including angiolymphatic invasion), higher mitotic rate, vertical growth phase, age $\leq 60$ years, Clark level greater than III, multiple nodal basins, axial location, and ulceration have been reported with conflicting results in association with a positive SLN in numerous series. ${ }^{2,8,13,14,18-23}$

With univariate analysis from our patients entered into a database, the following factors had a significant rela- 


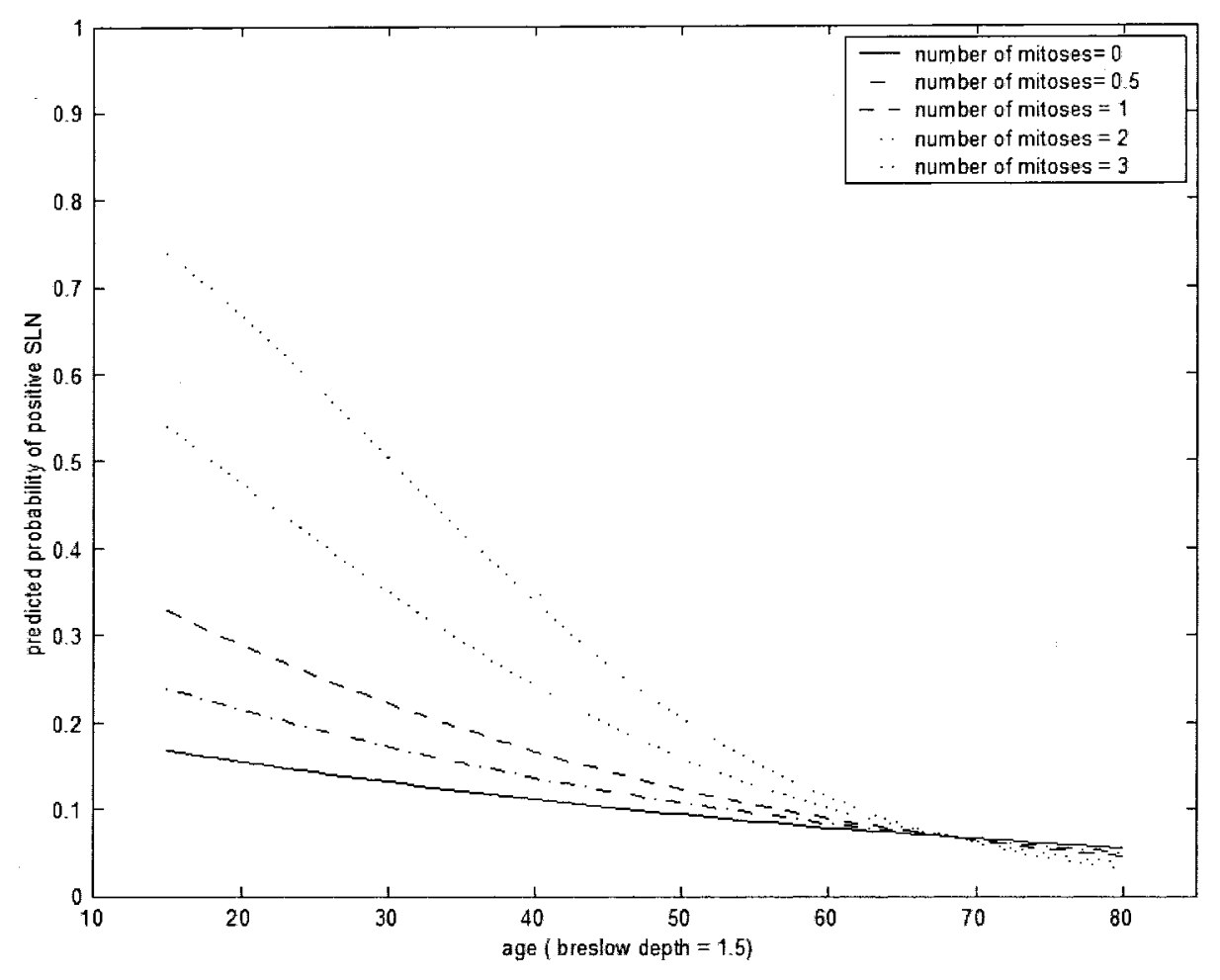

FIG. 4. The effect of mitotic rate on sentinel lymph node (SLN) positivity is lost with increasing age. A probability plot for a positive SLN for increasing age and mitotic rate for a $1.5-\mathrm{mm}$ Breslow depth melanoma demonstrates that mitotic rate loses its significance in predicting nodal positivity with increasing age. At 35 years of age or younger, there is marked separation of the curves for different mitotic rates, but by age 60 , the various curves have entirely converged. tionship with a positive SLN: number of mitoses $(P<$ $.0001)$, presence of ulceration $(P=.04)$, Breslow depth $(P<.0001)$, younger age $(P=.003)$, and upper extremity $(P=.02$, inverse correlation) or truncal $(P=.01)$ location. Multivariate analysis demonstrated that once age, number of mitoses, and Breslow depth were included, no other factor-including ulceration-was significantly associated with a positive SLN.

Our results stand in contrast to several other studies that have reported an association between the presence of ulceration and a positive SLN status. ${ }^{2,7,8,12,13,19-23}$ This difference may be due to differences in the diagnostic criteria used to define ulceration or to differences in the end point measured (H\&E-positive nodes vs. H\&Eand/or immunochemistry-positive nodes, as in our study), or they may be due to the number of histological features studied. The AJCC has defined ulceration broadly as the absence of an intact epidermis overlying the melanoma. Interpretation of ulceration, however, varies, even among dermatopathologists who specialize in melanoma. ${ }^{25}$ For example, lesions with exuberant scale crust and serum at the surface and a markedly attenuated epidermis may, in fact, have a thin rim of stratum corneum or parakeratotic scale at the surface. Some experts may regard this as a sign of ulceration, whereas others (including ourselves) do not. By standard convention, in the presence of ulceration, Breslow depth is obtained by measuring from the base of the ulcer to the deepest tumor extension. This potentially results in a thinner Breslow depth in ulcerated versus nonulcerated lesions, another factor that may in part explain an association between ulceration and a worse-than-expected prognosis for a given Breslow depth. At our institution, two measurements are routinely obtained if a lesion is ulcerated: one from the base of the ulcer and another in the deepest area of tumor extension overlying intact epidermis. Final assignment of depth is based on the deeper of the two measurements. This may represent another reason why, in our multivariate analysis, ulceration is not a significant factor.

Although variable criteria for the definition of ulceration may explain some of the differences seen in various published analyses, another part is the inclusion or exclusion of other factors in the multivariate analysis. Not all authors, for example, include the number of mitoses per square millimeter in their multivariate model. Without this factor, ulceration would have been considered a significant variable predicting for SLN positivity in our series. With age, ulceration, and mitotic rate, the odds ratio estimate for ulceration is 1.48 (95\% confidence interval, .81-2.70; $P=.21$ ). If mitotic rate is removed, then the odds ratio for ulceration is 2.01 (95\% confidence interval, $1.14-3.54 ; P=.02$ ). Clearly, given the prominence that ulceration now plays in the revised AJCC 


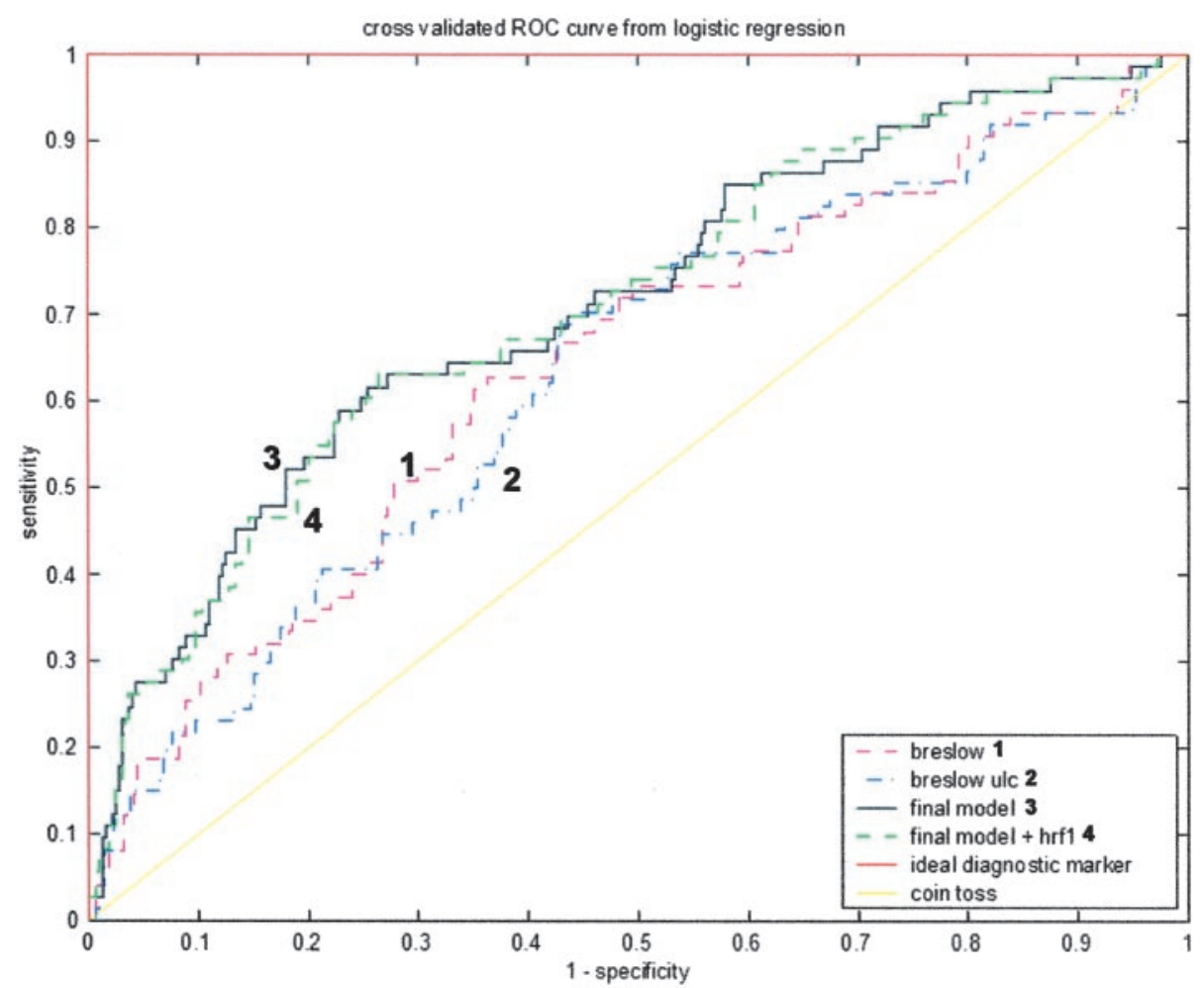

FIG. 5. Receiver operator characteristic (ROC) curves for the prediction of sentinel lymph node (SLN) positivity with different predictive models. Breslow thickness (curve 1) alone has the poorest sensitivity and specificity-little better than a coin toss. Breslow thickness plus ulceration (curve 2) does not increase the accuracy of prediction. The ROC curve for the predictive model of Breslow thickness, age, and mitotic rate (curve 3; solid line) moves up and to the left relative to the first two curves, indicating greater sensitivity and specificity. The fourth curve uses the total number of high-risk factors (hrf)—ulceration, high mitotic rate, microsatellitosis, angiolymphatic spread, vertical growth phase, and regression-but does not improve the shape of the ROC curve at all. staging system, further study is required to determine the optimal definition of ulceration, the proper method of assigning Breslow depth for ulcerated lesions, and the true independent prognostic significance of ulceration, particularly when mitotic rate is assessed.

Against this background, our own observations regarding the importance of age as a predictor for SLN positivity bear further elaboration. In melanoma, increasing age is generally thought of as a negative prognostic factor for survival, ${ }^{15}$ yet our results showed that decreasing age was associated with an increased likelihood of finding a positive node. Of course, this observation does not mean that stage for stage an older patient would fare better than a younger one. Our results highlight the important role of mitotic rate in younger patients; for melanoma patients 35 years of age or younger, increasing mitotic rate has a greater effect on the SLN positivity rate than increasing Breslow thickness. Extrapolation of our results also suggests that young patients with thin tumors $(<1 \mathrm{~mm})$ but high mitotic rates likely have an SLN positivity rate great enough to justify routine performance of SLN biopsy. However, only a prospective trial can determine how high the risk is and whether there is a thickness cutoff (e.g., $<.80 \mathrm{~mm}$, as recently proposed ${ }^{26}$ or a specific level of mitotic activity below which the likelihood of finding a positive SLN is too low to justify use of the procedure. Still, our results indicate how arbitrary and incomplete the decision-making process can be about suitability for SLN biopsy in thin melanomas, where traditionally only the presence of ulceration, regression, an involved deep margin, or-in some centers-Clark level IV or V invasion is used to select patients with thin melanomas for SLN biopsy. To our knowledge, no one has yet advocated selecting younger patients and, particularly, those with mitotically active thin melanomas for SLN biopsy.

Because many of the histological factors are correlated with each other, it is not surprising that different studies find slightly different sets of significant factors. The question left unanswered by all these studies is whether clinicopathologic factors can predict nodal positivity with sufficient accuracy to actually obviate the need to perform the diagnostic procedure. To evaluate this question directly, we analyzed ROC curves of our data set. The ROC analysis illustrates the sensitivity and specificity of using Breslow depth and other factors to predict SLN positivity. From the equations given, the predicted probability of a positive SLN can be calculated. A cutoff for the predicted probability is chosen, and this leads to a specificity and sensitivity. By changing the cutoff point, one can trade off specificity and sensitivity. Ideally, one would like both high specificity and high sen- 
sitivity. The actual values of sensitivity and specificity in the ROC curves are far from representing an ideal test. For example, if the multivariate model is used and the cutoff for predicting that an SLN will be positive is a prediction score (generated by the multivariate model's equation) of $>.191$, the prediction will be wrong for $39 \%$ (sensitivity) of the patients who are truly positive and for $28 \%$ (specificity) of the patients who are truly negative.

Although this level of predictive accuracy may be the best we can currently achieve with the available clinicopathologic features, these error rates indicate that there is still considerable variability in the likelihood of nodal metastases not accounted for by recognized clinicopathologic factors. The implication of this is clear: until and unless new predictive factors can be found, we will never be able to accurately determine who will have a positive SLN biopsy with sufficient accuracy to eliminate the need for the procedure.

\section{SUMMARY}

Factors identified as independent predictors of a positive SLN in our analysis were Breslow depth, number of mitoses per square millimeter, and younger age. Probabilistic models such as ours may ultimately find their greatest clinical use in identifying which patients with melanoma are at sufficient risk for lymph node metastasis to justify the risks of the procedure, rather than eliminating the need for the procedure itself.

\section{REFERENCES}

1. Morton DL, Thompson JF, Essner R, et al. Validation of the accuracy of intraoperative lymphatic mapping and sentinel lymphadenectomy for early-stage melanoma: a multicenter trial. Multicenter Selective Lymphadenectomy Trial Group. Ann Surg 1999; 230:453-63.

2. Gershenwald JE, Thompson W, Mansfield PF, et al. Multi-institutional melanoma lymphatic mapping experience: the prognostic value of sentinel lymph node status in 612 stage I or II melanoma patients. J Clin Oncol 1999;17:976-83.

3. Cherpelis BS, Haddad F, Messina J, et al. Sentinel lymph node micrometastasis and other histologic factors that predict outcome in patients with thicker melanomas. J Am Acad Dermatol 2001; $44: 762-6$.

4. Thompson JF, Uren RF, Shaw HM, et al. Location of sentinel lymph nodes in patients with cutaneous melanoma: new insights into lymphatic anatomy. J Am Coll Surg 1999;189:195-204.

5. Morton DL, Wen DR, Wong JH, et al. Technical details of intraoperative lymphatic mapping for early stage melanoma. Arch Surg 1992;127:392-9.

6. McMasters KM, Reintgen DS, Ross MI, et al. Sentinel lymph node biopsy for melanoma: controversy despite widespread agreement. J Clin Oncol 2001;19:2851-5.

7. Cascinelli N, Belli F, Santinami M, et al. Sentinel lymph node biopsy in cutaneous melanoma: the WHO Melanoma Program experience. Ann Surg Oncol 2000;7:469-74.

8. Mraz-Gernhard S, Sagebiel RW, Kashani-Sabet M, Miller JR III, Leong SP. Prediction of sentinel lymph node micrometastasis by histological features in primary cutaneous malignant melanoma. Arch Dermatol 1998;134:983-7.

9. Belli F, Lenisa L, Clemente C, et al. Sentinel node biopsy and selective dissection for melanoma nodal metastases. Tumori 1998; 84:24-8.

10. Lenisa L, Santinami M, Belli F, et al. Sentinel node biopsy and selective lymph node dissection in cutaneous melanoma patients. J Exp Clin Cancer Res 1999;18:69-74.

11. Gershenwald JE, Mansfield PF, Lee JE, Ross MI. Role for lymphatic mapping and sentinel lymph node biopsy in patients with thick $(\geq 4 \mathrm{~mm})$ primary melanoma. Ann Surg Oncol 2000;7:160-5.

12. Essner R, Conforti A, Kelley MC, et al. Efficacy of lymphatic mapping, sentinel lymphadenectomy, and selective complete lymph node dissection as a therapeutic procedure for early-stage melanoma. Ann Surg Oncol 1999;6:442-9.

13. Nguyen CL, McClay EF, Cole DJ, et al. Melanoma thickness and histology predict sentinel lymph node status. Am J Surg 2001;181: $8-11$.

14. McMasters KM, Wong SL, Edwards MJ, et al. Factors that predict the presence of sentinel lymph node metastasis in patients with melanoma. Surgery 2001;130:151-6.

15. Balch CM, Soong S-J, Gershenwald JE, et al. Prognostic factors analysis of 17,600 melanoma patients: validation of the American Joint Committee on Cancer melanoma staging system. J Clin Oncol 2001;19:3622-34.

16. Clary BM, Brady MS, Lewis JJ, Coit DG. Sentinel lymph node biopsy in the management of patients with primary cutaneous melanoma: review of a large single-institutional experience with an emphasis on recurrence. Ann Surg 2001;233:250-8.

17. Joseph E, Brobeil A, Glass F, et al. Results of complete lymph node dissection in 83 melanoma patients with positive sentinel nodes. Ann Surg Oncol 1998;5:119-25.

18. Bedrosian I, Faries MB, Guerry D IV, et al. Incidence of sentinel node metastasis in patients with thin primary melanoma $(\leq 1 \mathrm{~mm})$ with vertical growth phase. Ann Surg Oncol 2000;7:262-7.

19. Sabel MS, Gibbs JF, Cheney R, McKinley BP, Lee JS, Kraybill WG. Evolution of sentinel lymph node biopsy for melanoma at a National Cancer Institute-designated cancer center. Surgery 2000; 128:556-63.

20. Wagner JD, Gordon MS, Chuang TY, et al. Predicting sentinel and residual lymph node basin disease after sentinel lymph node biopsy for melanoma. Cancer 2000;89:453-62.

21. Jansen L, Nieweg OE, Peterse JL, Hoefnagel CA, Olmos RAV, Kroon BBR. Reliability of sentinel lymph node biopsy for staging melanoma. Br J Surg 2000;87:484-9.

22. Gershenwald JE, Tseng CH, Thompson W, et al. Improved sentinel lymph node localization in patients with primary melanoma with the use of radiolabeled colloid. Surgery 1998;124:203-10.

23. Porter GA, Ross MI, Berman RS, Lee JE, Mansfield PF, Gershenwald JE. Significance of multiple nodal basin drainage in truncal melanoma patients undergoing sentinel lymph node biopsy. Ann Surg Oncol 2000;7:256-61.

24. Karimipour DJ, Lowe L, Su L, et al. Standard immunostains for melanoma in sentinel lymph node specimens: which ones are most useful? J Am Acad Dermatol (in press).

25. Spatz A, Cook MG, Elder DE, Piepkorn M, Ruiter DJ, Barnhill RL. Interobserver reproducibility of ulceration assessment in primary cutaneous melanomas. Eur J Cancer 2003;39:1861-5.

26. Zapas JL, Coley HC, Beam SL, Brown SD, Jablonski KA, Elias EG. The risk of regional lymph node metastases in patients with melanoma less than $1.0 \mathrm{~mm}$ thick: recommendations for sentinel lymph node biopsy. J Am Coll Surg 2003;197:403-7. 